\title{
Traceroute-based route gathering and repairing mechanism of client-independent IP geolocation system
}

\author{
Yongcai Tao, Weiwei Jia, Lei Shi \\ School of Information Engineering, \\ Zhengzhou University, \\ Zhengzhou, China \\ ieyctao@zzu.edu.cn
}

\begin{abstract}
Delay measurement-based IP Geolocation can gain better IP geolocation performance. However, there are still some defects needed to be improved, including inaccurate landmark information, incomplete traceroute information and inaccurate traceroute repairing information. To address the issues, the paper proposes a traceroute-based route gathering and repairing mechanism of client-independent IP geolocation system. The contributions include: 1) a landmark information filtering mechanism is presented to filter the noise data; 2) an Improved TraceRoute Gathering algorithm (ITRG) is proposed to increase the integrity of route information; 3) a Reverse Route Delay Repairing algorithm (RRDR) is presented to solve the problem of route inflation, guaranteeing that the delay value of current router is smaller than that of its next router. The experimental results show that using the improved IP geolocation mechanism proposed in the paper, the median error distance of IP geolocation is reduced to $5.42 \mathrm{~km}$ from $9.60 \mathrm{~km}$.
\end{abstract}

Keywords- IP Geolocation; traceroute; route inflation; TCP; ICMP

\section{INTRODUCTION}

With the rapid development of Internet and emergence of new Internet applications, IP geolocation service is playing an increasingly important role in a number of Internet applications such as network management, online advertising, cloud computing and etc.[1,2,10]

IP geolocation can be classified into two categories: client-dependent system and client-independent system. Though client-dependent systems can provide highly accurate IP geolocation inferences, now, many Internet applications such as location-based access restrictions, context-aware security, and online advertising, can not depend on client-dependent IP geolocation service. So, a highly accurate client-independent geolocation system becomes an important goal for the Internet. One example is that with the IP geolocation service, online advertising can provide more accurate and timely advertising services for users. Another example is that to improve the scalability, availability and energy efficiency of cloud computing system, a highly accurate client-independent geolocation system can help select a properly dispersed set of resource nodes within a cloud system.

In recent years, the client-independent IP geolocation has attracted many researches. Among these researches, Wang et al. [3] propose a novel client-independent geolocation: street-

\author{
Lin Wei, Yangjie Cao \\ School of Software, \\ Zhengzhou University, \\ Zhengzhou, China
}

level client-independent IP geolocation system, which includes five parts: gathering landmarks, getting traceroute paths, repairing traceroute results, generating "LandmarkRouter" database and gaining target IP geolocation. The system [3] can geolocate IP address with a median error distance of 690 meters in an academic environment, and improve the median accuracy by 50 times relative to [1] and by approximately 100 times relative to [2].

However, there are some defects in the system proposed by Wang et al. [3]

1) Inaccurate landmark information. Some landmark nodes can not reflect their real geographic information.

2) Incomplete traceroute information. The system only sends TCP and ICMP messages during getting traceroute information.

3) Inaccurate traceroute repairing information. This is associated with the route inflation, which leads to the fact that the delay value of current hop is larger than that of its next hops.

Route inflation is mainly caused by the heterogeneity of Autonomous System (AS), the limitation of existing routing protocols and the irregular eruption of network traffic.

To address the issue of route inflation, the paper proposes a traceroute-based route gathering and repairing mechanism of client-independent IP geolocation system. The contributions lie in the followings.

1) A landmark information filtering mechanism is presented to filter the noise data by comparing the network distance and physical distance of landmarks.

2) An Improved TraceRoute Gathering algorithm (ITRG) is proposed to increase the integrity of route information. ITRG distributes three kinds of packets: TCP, ICMP and UDP. It selects the longest route path information which can reach the target IP node, and replaces the route delay using the minimum delay of other two route information. Finally, the network delay between any two neighbor routers is minimal.

3) A Reverse Route Delay Repairing algorithm (RRDR) is presented to solve the problem of route inflation, and to guarantee the delay value of the current router is smaller than that of its next router.

\section{RELATED WORK}

There have been great deals of researches on both clientdependent and client-independent IP geolocation. 
In current client-dependent IP geolocation systems, there are many mature technologies, such as GPS-based geolocation, cell tower and Wi-Fi-based geolocation [4, 5] and W3C geolocation [6], which can provide accurate results, e.g., 200-1000 meters accuracy in cell tower scenario, and 10-20 meters in Wi-Fi scenario. However, these technologies all need the clients' support for geolocation.

The researches on client-independent IP geolocation mainly focus on data mining-based and delay measurementbased geolocation [1-3,7,8]. There have been some achievements on delay measurement-based IP geolocation. Padmanabhan et al. [7] design GeoPing geolocation system which assumes that two machines that have same delay vectors tend to be close to each other. Gueye et al. [8] introduce Constraint Based Geolocation mechanism (CBG), a method that provides a continuous geo space by using multilateration with distance constraints. Katz-Bassett et al. [2] present Topology Based Geolocation (TBG) based on the fact that routers close to the targets can be more accurately located. Wong et al. [1] propose Octant, which considers the locations of intermediate routers as landmarks to geolocate the target. Wang et al. [3] propose a street-level clientindependent IP geolocation. The methodology consists of two powerful components. First, it utilizes a system that effectively harvests geolocation information available on the Web to build a database of landmarks in a given ZIP code. Second, it employs a three tiered system that begins at a large, coarse-grained scale and progressively works its way to a finer, street-level scale. The system [3] can geolocate IP addresses with a median error distance of 690 meters in an academic environment, and improve the median accuracy by 50 times relative to [1] and by approximately 100 times relative to [2].

\section{BACKGROUND}

Since our work is based on the street-level clientindependent IP geolocation system proposed by Wang et al. [3], the paper would briefly review the IP geolocation mechanism.

The street-level client-independent IP geolocation system [3] includes five steps: gathering landmarks, getting traceroute paths, repairing traceroute results, generating "Landmark-Router" database and gaining target IP geolocation. The system architecture is shown in Figure 1. The working process is as follows.

1) Gathering landmarks. To guarantee the accuracy of IP geolocation, a certain number of landmarks, which are mainly the Web servers of school, hospital, government, supermarket, and so on, should be extracted.

2) Getting traceroute paths. The system uses a certain number of machines as vantage points to distribute TCP and ICMP packets to the above-mentioned landmarks.

3) Repairing traceroute results. In step 2, there exits one issue: route inflation, which means that the delay value of current hop is larger than that of its next hop, the system modify the delay value of current hop to be the one of its next hop.
4) Generating "Landmark-Router" database. Through steps 1,2 and 3 , the system generates the mapping database of landmarks and routers.

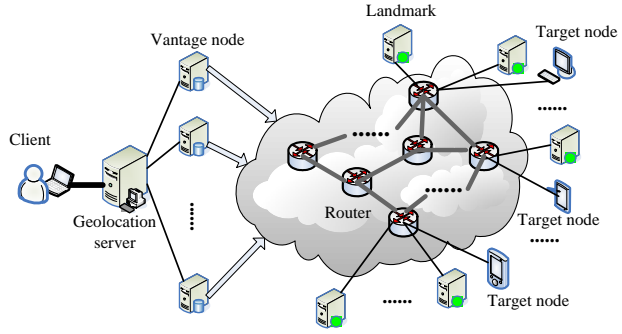

Figure 1. IP geolocation system architecture

5) Gaining target IP geolocation. When a target IP need be geolocated, the vantage points send traceroute packets to the target IP to get their traceroute information. Then through comparing the traceroute information with the LandmarkRouter database, the system fetches all shared routers, and computes the relative delays which go through the shared routers between the target IP and landmarks. Finally, the system gets the landmark which has the shortest delay with the target IP and sends the address information to the client.

However, there are still some defects needed to be improved in the IP geolocation system proposed by Wang et al. [3], including inaccurate landmark information, incomplete traceroute information and inaccurate traceroute repairing information.

\section{LANDMARK INFORMATION FILTERING MECHANISM}

Landmark information is one of key factors of IP geolocation system. However, some landmark information can not reflect their real geographic information. For example, the geographic information of landmarks is gained from Web page, but their servers are located in other places rather than the places that the Web page indicated. Or the servers of landmarks are moved to other places, but the relative information doesn't be updated in time.

To solve the issue, a landmark information filtering mechanism is presented to filter the inaccurate landmark information. The rationales and workflow are as follows.

1) The landmark nodes are classified into different groups in which the nodes have the same "edge router". Edge router is the router located at the fringe of network and has little input load and the delay is low, which can degrade the influence of traceroute inflation.

2) In each group, the network distance and physical distance between any two landmark nodes are calculated. If the network distance between two landmark nodes is larger than their physical distance, or the network distance between two landmark nodes is less than the set value, the landmarks' information can be regarded to be accurate, whereas to be fault.

The network distance between two nodes is calculated by multiplying packet traveling delay by packet traveling speed. In the paper, we adopt 4/9 c from [3] as the packet traveling speed, c denotes the speed of light in a vacuum. 
The physical distance between two nodes is calculated by their latitude and longitude which can be obtained from landmark database. The physical distance between two landmark nodes L1 and L2 (PD(L1,L2)) can be calculated according to formula 1 . In formula 1 , lat1 and lat2 represent the latitude of L1 and L2, lon1 and lon2 represent the longitude of L1 and L2.

$$
\begin{aligned}
\mathrm{PD}(\mathrm{L} 1, \mathrm{~L} 2)= & \mathrm{ACOS}(\mathrm{SIN}(\mathrm{lat} 1) * \mathrm{SIN}(\text { lat2)+ } \\
& \mathrm{COS}(\text { lat1) } * \operatorname{COS}(\text { lat2) } * \operatorname{COS}(\operatorname{lon} 2-\operatorname{lon} 1)) * R
\end{aligned}
$$

The formula 1 is a transformation of arc length formula $(\mathrm{L}=\alpha * \mathrm{R})$ which is used to calculate the distance between two points on earth, a represents the central angle of the arc between $\mathrm{L} 1$ and $\mathrm{L} 2, \mathrm{R}$ represents radius of the earth.

The ideas are that if two landmark nodes are both connected directly to the same "edge router", their network distance would be larger than their physical distance, or their network distance would be lower than the set value (such as $80 \mathrm{~km}$, the diameter of one medium-sized city usually is about $80 \mathrm{~km}$ ).

Through the landmark information filtering mechanism, the system can gain accurate landmark information which is the foundation of IP geolocation.

\section{Improved TraceRoute Gathering ALgORITHM}

\begin{tabular}{|c|c|}
\hline Symbol & Meaning \\
\hline Landmark[] & $\begin{array}{l}\text { landmark set including a number of } \\
\text { landmark nodes }\end{array}$ \\
\hline Vantage[] & $\begin{array}{l}\text { vantage set including a number of } \\
\text { vantage nodes }\end{array}$ \\
\hline TraInfo[i][k] & $\begin{array}{l}\text { traceroute information with max } \\
\text { path length of landmark } i \text { in the kth } \\
\text { round }\end{array}$ \\
\hline TraInfo[i] & $\begin{array}{l}\text { traceroute information with max } \\
\text { path length and minimum delay of } \\
\text { landmark } \mathrm{i}\end{array}$ \\
\hline T_path[i][j] & $\begin{array}{l}\text { traceroute path information of TCP } \\
\text { from vantage } \mathrm{j} \text { to landmark } \mathrm{i}\end{array}$ \\
\hline I_path[i][j] & $\begin{array}{c}\text { traceroute path information of } \\
\text { ICMP from vantage j to landmark i }\end{array}$ \\
\hline U_path[i][j] & $\begin{array}{l}\text { traceroute path information of UDP } \\
\text { from vantage } \mathrm{j} \text { to landmark } \mathrm{i}\end{array}$ \\
\hline T_path[i] & $\begin{array}{l}\text { TCP traceroute information with } \\
\text { max path length and minimum delay of } \\
\text { landmark i }\end{array}$ \\
\hline I_path[i] & $\begin{array}{l}\text { ICMP traceroute information with } \\
\text { max path length and minimum delay of } \\
\text { landmark i }\end{array}$ \\
\hline U_path[i] & $\begin{array}{l}\text { UDP traceroute information with } \\
\text { max path length and minimum delay of } \\
\text { landmark i }\end{array}$ \\
\hline DelayH[i] & delay value of the ith hop \\
\hline
\end{tabular}

TABLE I. SYMBOLS DEFINITION

The IP geolocation system is based on the path information gathered by traceroute, so the integrity, diversity and accuracy of traceroute information will badly affect the accuracy of IP geolocation. In order to provide more accurate and abundant traceroute path information, the traceroute gathering algorithm should meet the following requirements: 1) Getting the path information that can reach the landmark; 2) Selecting the longest path information which has the most routers; 3) Obtaining the optimal delay (namely, the minimum delay) of each router.

To address the issue of incomplete traceroute information in the system in [3], the paper proposes an Improved TraceRoute Gathering algorithm (ITRG), which is shown in Algorithm 1. The symbols used in the paper are listed in TABLE I .

The rationale of ITRG is as follows. First, the system sends packets of TCP, ICMP and UDP to the landmarks via vantage nodes, and then retrieves the corresponding traceroute path information (lines 1-7). Second, the system selects the traceroute information with max length path from the three types (TCP, ICMP and UDP) of path information. The system prefers the path information which can reach the landmarks and have more abundant router information. Finally, using the information of other two paths, the system updates the selected path (lines 8-19). This means that for the delay of the same router on three paths from the same vantage point, the system takes the minimum one to minimize the impact of network delays.

Algorithm 1: Improved TraceRoute Gatherring algorithm

Input: Landmark[], Vantage[];

(ITRG)

Output: TraInfo[][];

1. for each landmark $i \in$ Landmark[] do

2. for each vantage $\mathrm{j} \in$ Vantage[] do

3. $\quad$ send TCP, ICMP and UDP packets to

landmark i from vantage $\mathrm{j}$;

4. $\quad$ T_path[i][j]=traceroute(TCP, landmark i);

5. $\quad$ I_path[i][j] =traceroute(ICMP, landmark i);

6. $\quad$ U_path $[\mathrm{i}][\mathrm{j}]=$ traceroute(ICMP, landmark i);

7. endfor

8. $\quad$ T_path $[\mathrm{i}]=$ traceroute information with $\max$ TCP path length and minimum delay of landmark $i$;

9. $\quad$ I_path $[\mathrm{i}]=$ traceroute information with $\max$ ICMP path length and minimum delay of landmark i;

10. U_path $[\mathrm{i}]=$ traceroute information with max UDP path length and minimum delay of landmark i;

11. if all packets reach landmark i then

12. TraInfo[i][k]=max length one of (T_path[i], I_path[i], U_path[i]);

update TraInfo[i] [k] using the left two;

13. elseif any two of (T_path[i], I_path[i],

14. elseif any two of (1_path[i], I_path[i],

15. TraInfo[i][k]=max length one of the two;

16. update TraInfo[i][k] using the left two;

17. elseif only one path reaches landmark i then

18. $\quad$ TraInfo[i] $[\mathrm{k}]=$ the reached one;

19. update TraInfo[i][k] using the left two;

$20 . \quad$ else

21. TraInfo[i][k]=NULL;

22. endif

23. endfor 


\section{ReVERse Route Delay RePairing Algorithm}

As network is heterogeneous and the congestion reasons are diverse, the route delay may not always be accurate and has great impact on IP geolocation.

ITRG repairs the route delay by obtaining the minimum delay in one round traceroute. The paper introduces a Reverse Route Delay Repairing algorithm (RRDR) to implement additional repairing of the route information in two aspects: First, RRDR combines and updates the route information by obtaining the minimum delay among multiple rounds of traceroute; Second, RRDR solves the route inflation using the reverse repairing mechanism.

\section{A. Repairing Route Information among Multiple Rounds of} Traceroute

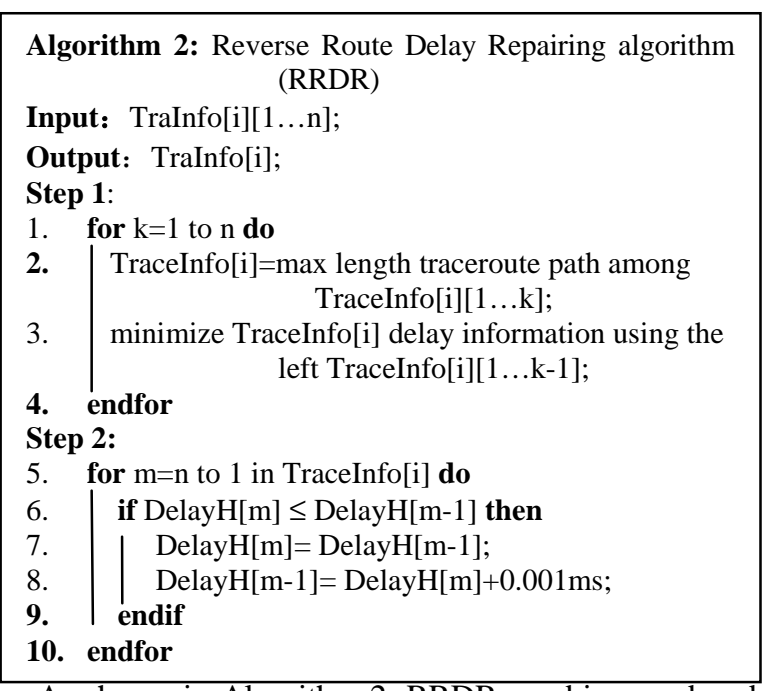

As shown in Algorithm 2, RRDR combines and updates the route information in Step 1 (lines 1-4). First, resemble to ITRG (Algorithm 1), RRDR obtains the max length path by combining multiple rounds of traceroute. Then, RRDR minimizes the delay information of each hop using the left traceroute information in Step 2 (lines 5-9).

\section{B. Reverse Route Delay Repairing}

In the algorithm proposed in [3], if the delay of current hop is larger than that of its next hop, it will be replaced to be its next hop. This repairing mechanism has two issues: First, the delay between two routers (or between router and landmark) may be $0 \mathrm{~ms}$. Second, when using the information to build "Landmark-Router" database, it is difficult to bind the best data.

To address the above issues, Algorithm 2 amends the delay value of current hop to be a little larger than that of its next hop (e.g. $0.001 \mathrm{~ms}$ ) while dealing with the route inflation until the delay of each hop is shorter than that of its next hop. Thus, it can ensure that not only the delay is accurate, but also the delay between two routers is not $0 \mathrm{~ms}$.

\section{PERFORMANCE EVALUATION}

\section{A. Experimental Environment}

The IP geolocation system architecture is shown in Figure 1. The IP Geolocation server runs the RRDR algorithm and is located at Amazon EC2. Its configuration information is 6.5 Intel dual-core CPU $3.25 \mathrm{GHz}$ and $18 \mathrm{G}$ Memory. The vantage nodes run the ITRG algorithm, and include 30 rented nodes distributed at over 30 American cities (Seattle, Los Angeles, Miami, etc.) and 130 PlanetLab [9] nodes at US research institutions. The configuration information of rented machines is Intel dual-core CPU 2.7 $\mathrm{GHz}$, memory 2G. The configuration of PlanetLab [9] nodes is Intel dual-core CPU $2.3 \mathrm{GHz}$, memory $4 \mathrm{G}$.

\section{B. Experimental Results}

We conduct the experiments by comparing the IP geolocation algorithms in [3] (called original algorithm) and ITRG and RRDR.

The experiment is based on two datasets:

Dataset 1: the 5 rounds traceroute results of 15616 landmarks on 30 rented servers.

Dataset 2: the 2 rounds traceroute results of 15616 landmarks on 130 PlanetLab servers [9].

1) ITRG

TABLE II shows the comparison of traceroute path between the original algorithm [3] and ITRG. In TABLE II, L_T_R(1-5) represents the landmark traceroute information of the round (1-5) of dataset 1 . L_T_PL(1-2) represents the landmark traceroute information of the round (1-2) of dataset 2. As shown in TABLE II, the results show that the number of valid paths is increased significantly by ITRG. In particular, the number of valid paths in round 4 is increased by $50.4 \%$.

To increase the variety of traceroute information, ITRG not only adds UDP packet, but also improves path selection mechanism. Using the IP geolocation with ITRG, the median error distance is reduced to $6.41 \mathrm{~km}$ from $9.60 \mathrm{~km}$ compared with the original algorithm [3], as shown in Figure 2.

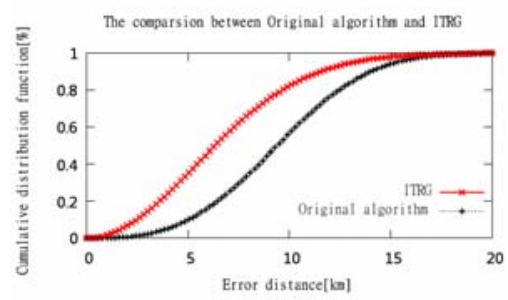

Figure 2. The comparison of the original algorithm [3] and ITRG

\section{2) $R R D R$}

TABLE III shows the comparison of combining results of traceroute path of multi rounds. In TABLE III, Combine() represents the combining function of traceroute path of multi rounds. TABLE III shows that we can get better path information by RRDR. In particular, regarding growth rate, we find that after combining the traceroute paths among 5 rounds, it becomes as low as $1.1 \%$. Moreover, we observe a clear 'drop' of the growth rate between each round. Also, 
regarding update rate, we see the similar trends of 'drop' between each round, and the data we collected is getting optimal.

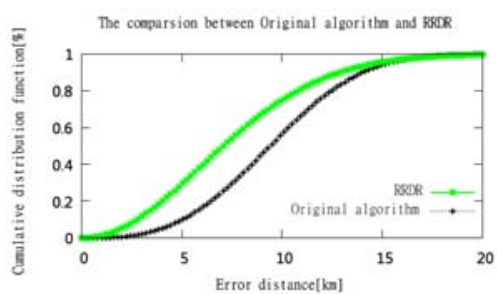

Figure 3. The comparison between original algorithm and RRDR

RRDR tries to degrade the bad effects of route inflation. Using the IP geolocation with RRDR, the median error distance is reduced to $7.22 \mathrm{~km}$ from $9.60 \mathrm{~km}$ compared with the original algorithm [3], as shown in Figure 3.

To sum up, using the IP geolocation with ITRG and RRDR, the median error distance is reduced to $5.42 \mathrm{~km}$ from $9.60 \mathrm{~km}$ compared with the original algorithm [3], as shown in Figure 4.

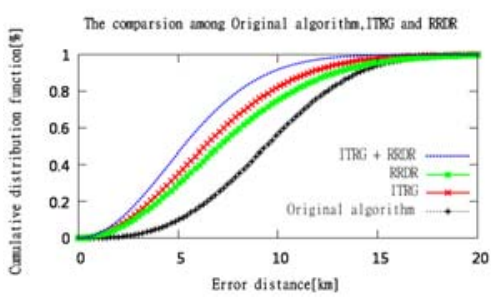

Figure 4. The comparison among original algorithm, ITRG and RRDR

TABLE II. COMPARISON OF TRACEROUTE PATH OF ORIGINAL ALGORITHM AND ITRG

\begin{tabular}{|c|c|c|c|c|c|}
\hline & $\begin{array}{c}\text { No. of paths } \\
\text { (Original algorithm) }\end{array}$ & $\begin{array}{c}\text { No. of valid paths } \\
\text { (Original algorithm) }\end{array}$ & $\begin{array}{c}\text { No. of paths } \\
\text { (ITRG) }\end{array}$ & $\begin{array}{c}\text { No. of valid paths } \\
\text { (ITRG) }\end{array}$ & Increased rate of valid paths \\
\hline L_T_R1 & 302984 & 299954 & 435785 & 430120 & $43.4 \%$ \\
\hline L_T_R2 & 282704 & 279312 & 422080 & 417859 & $49.6 \%$ \\
\hline L_T_R3 & 297298 & 294622 & 428225 & 424799 & $44.2 \%$ \\
\hline L_T_R4 & 255010 & 251440 & 383967 & 378208 & $50.4 \%$ \\
\hline L_T_R5 & 255999 & 254207 & 376069 & 369300 & $45.3 \%$ \\
\hline L_T_PL1 & 462150 & 457066 & 693217 & 686285 & $50.2 \%$ \\
\hline L_T_PL2 & 445332 & 440880 & 674640 & 665870 & $51.0 \%$ \\
\hline
\end{tabular}

TABLE III. COMBINING RESULTS OF TRACEROUTE PATH OF MULTI ROUNDS

\begin{tabular}{|l|c|c|c|}
\hline & Number of valid paths & $\begin{array}{c}\text { Increased rate of } \\
\text { paths }\end{array}$ & Update rate of paths \\
\hline L_T_R1 & 430120 & $0 \%$ & $0 \%$ \\
\hline Combine(R1,R2) & 459701 & $6.8 \%$ & $39.2 \%$ \\
\hline Combine(R1,R2,R3) & 475050 & $3.3 \%$ & $30.3 \%$ \\
\hline Combine(R1,R2,R3,R4) & 490496 & $3.2 \%$ & $23.0 \%$ \\
\hline Combine(R1,R2,R3,R4,R5) & 495778 & $1.1 \%$ & $12.1 \%$ \\
\hline L_T_PL1 & 457066 & $0 \%$ & $0 \%$ \\
\hline Combine(PL1,PL2) & 509976 & $11.6 \%$ & $35.6 \%$ \\
\hline
\end{tabular}

\section{CONCLUSIONS}

Existing IP Geolocation System only sends TCP and ICMP packets while gathering landmarks, thus we can't get a large variety of landmarks. Moreover, some landmarks information is fault and the repairing of route inflation is not complete. To solve the issues, the paper presents: 1) a landmark information filtering mechanism to filter the inaccurate landmark information, 2) ITRG to increase the variety of the traceroute information, and 3) RRDR to select the best traceroute information among multiple rounds.

Using the improved IP geolocation mechanism proposed in the paper, the median error distance of IP geolocation is further reduced.

\section{REFERENCES}

[1] B. Wong, I. Stoyanov, E. G. Sirer. Octant: a comprehensive framework for the geolocalization of Internet hosts. In Proc. of the 4th USENIX Symposium on Networked Systems Design \& Implementation (NSDI'07), 2007, Cambridge, MA, pp.313-326.

[2] E. Katz-Bassett, J. P. John, A. Krishnamurthy, D. Wetherall, T. Anderson, Y. Chawathe. Towards ip geolocation using delay and topology measurements. In Proc. of the 6th ACM SIGCOMM conference on Internet measurement (IMC’06), 2006, USA, pp.71-84.

[3] Y. Wang, D. Burgener, M. Flores, A. Kuzmanovic, C. Huang. Towards street-level client-independent ip geolocation. In Proc. of the 8th USENIX Symposium on Networked Systems Design \& Implementation (NSDI'11), 2011, Boston, MA, 5(5).

[4] Google maps with my location. http://www.google.com/mobile/gmm/mylocation/index.html. 2012.

[5] Skyhook. http://www.skyhookwireless.com/. 2012.

[6] Geolocation api specification. http://dev.w3.org/geo/api/specsource.html. 2012.

[7] V. N. Padmanabhan, L. Subramanian. An investigation of geographic mapping techniques for Internet host. In Proc. of the 2001 conference on Applications, technologies, architectures, and protocols for computer communications ( SIGCOMM '01), USA, 2001, pp.173185.(10)

[8] B. Gueye, A. Ziviani, M. Crovella, S. Fdida. Constraint-based geolocation of Internet hosts. IEEE/ACM Transactions on Networking , 2006, 14(6):1219-1232.

[9] PlanetLab. http://www.planet-lab.org/. 2012.

[10] D. Li, J. Chen, C. Guo, Y. Liu, J. Zhang, Z. Zhang, Y. Zhang. IPGeolocation mapping for moderately-connected Internet regions. IEEE Transaction on Parallel and Distributed Systems, 2012. 